\title{
Prognostic Value of the 24-Hour Neurological Examination in Anterior Circulation Ischemic Stroke: A post hoc Analysis of Two Randomized Controlled Stroke Trials
}

\author{
Srikant Rangaraju ${ }^{a} \quad$ Michael Frankel $^{a} \quad$ Tudor G. Jovin ${ }^{b}$ \\ ${ }^{a}$ Emory University, Atlanta, Ga., and b University of Pittsburgh Medical Center, \\ Pittsburgh, Pa., USA
}

\section{Key Words}

Ischemic stroke · Outcome · Prognosis · National Institute of Neurological Diseases and Stroke $\cdot$ Clinical examination

\begin{abstract}
Background: Early prognostication of long-term outcomes following ischemic stroke can facilitate medical decision-making. We hypothesized that the 24-hour National Institute of Health Stroke Scale (NIHSS) predicts 3-month clinical outcomes in anterior circulation stroke. Methods: Secondary analyses of the Interventional Management of Stroke 3 (IMS3) and intravenous tissue plasminogen activator (IV tPA) for acute ischemic stroke [National Institute of Neurological Diseases and Stroke (NINDS) IV tPA] trials were performed. In participants with documented 24-hour NIHSS and 3-month Modified Rankin Scale (mRS), the predictive power of the 24-hour NIHSS and 24-hour NIHSS improvement for 3-month outcomes [mRS $0-2$ and Barthel Index (BI) $\geq 95$ ] was assessed. Percentages of good outcome (mRS 0-2 or BI $\geq 95)$ at 3,6 , and 12 months and mean quality of life (EQ5DTM) index at 3 months across 24hour NIHSS quartiles were compared. Results: The majority of the study participants were included (IMS3 $n=587 / 656$, NINDS IV tPA $n=619 / 624$ ). The 24-hour NIHSS was correlated with 3-month $\mathrm{mRS}(\mathrm{R}=0.73)$ with excellent predictive power for mRS $0-2$ [area under the curve $(A \cup C)=0.91$ ] and $B I \geq 95$ (AUC $=0.9)$ in both cohorts. A model with the 24-hour NIHSS alone correctly classified $82-84 \%$ of patients in both cohorts. The percentages of good outcomes at 3-12 months across 24-hour NIHSS quartiles were similar in both cohorts. mRS 0-2 was achieved by $75.6-77.7 \%$ of patients with 24 -hour NIHSS $\leq 11$ but by only $1.4-3.6 \%$ with 24 -hour NIHSS $\geq 20$. The EQ5D index at 3 months varied among NIHSS $0-4$ (mean $0.86 \pm 0.16$ ), 5-11 (0.77 \pm 0.18$)$, and 12-19 (0.59 \pm 0.26$)$ quartiles. Conclusions: The 24-hour NIHSS strongly predicts long-term stroke outcomes and is associated with quality of life. Its easy availability, reliability, and validity support its use as an early prognostic marker and surrogate of clinical outcome in ischemic stroke.




\section{Introduction}

Anterior circulation ischemic stroke is associated with significant disability and variability in observed clinical outcomes [1]. Robust predictors of clinical outcome including age, medical comorbidities (diabetes, hypertension, atrial fibrillation), time to treatment, infarct size, and hemorrhagic transformation may explain some of this variability in clinical outcomes [2-4]. The importance of the neurological examination at $24-48 \mathrm{~h}$ after presentation in neurologically critically ill patients is well known to neurologists. A National Institute of Health Stroke Scale (NIHSS) score $\leq 2$ at 24 h strongly predicts the effectiveness of thrombolytic therapy [5], but data regarding its prognostic power for predicting long-term clinical outcome in ischemic stroke patients are limited [6,7]. In stroke patients, the NIHSS score is a wellaccepted and widely used scoring scheme that captures neurological deficits [8-10]. NIHSS on admission, while being independently associated with clinical outcomes in stroke patients, often changes within the first $24 \mathrm{~h}$ [6]. Early neurological improvement, a predictor of 3-month outcomes, might reflect timely recanalization of the occluded vessel [7], either spontaneously or due to treatment, while clinical decline may result from infarct expansion, stroke-related complications, cerebral edema or hemorrhage [11]. Since the follow-up neurological examination is likely influenced by a multitude of measured and unmeasured factors that may or may not be directly related to stroke, it is possible that this easily available yet complex variable by itself may have a significant prognostic value as a determinant of long-term clinical outcome.

We hypothesized that the 24-hour NIHSS accurately predicts 3-month clinical outcome in anterior circulation ischemic stroke patients. To test this hypothesis, we first performed a secondary analysis of the Interventional Management of Stroke 3 (IMS3) clinical trial database that compared intravenous tissue plasminogen activator (IV tPA) alone with IV tPA plus endovascular treatment in acute ischemic stroke patients [12]. The predictive power of the 24-hour NIHSS, absolute and relative improvements in NIHSS over $24 \mathrm{~h}$, and 3-month good outcomes [Modified Rankin Scale (mRS) score 0-2 and Barthel Index (BI) $\geq 95$ ] were determined. The additional prognostic value of the 24-hour NIHSS, after adjusting for age, medical comorbidities, and time to treatment, was also assessed. Risk categories based on observed rates of good outcome across 24-hour NIHSS quartiles were created. The predictive power of the 24-hour NIHSS and the identified risk strata were then validated in the National Institute of Neurological Diseases and Stroke (NINDS) IV tPA for acute ischemic stroke trial cohort.

\section{Materials and Methods}

\section{Patient Selection}

IMS3 was a multicenter, double-arm study evaluating clinical outcomes after IV tPA alone versus IV tPA plus endovascular therapy in patients with acute ischemic stroke. The study included subjects aged 18-82 years with initiation of IV tPA within $3 \mathrm{~h}$ of symptom onset and an NIHSS $\geq 10$ or $\geq 8$ points with the level of occlusion confirmed by CT angiography, at the onset of IV tPA. The validation population was derived from the NINDS IV tPA trial, a multicenter randomized clinical trial that compared IV tPA with placebo, for patients presenting with stroke symptoms measurable by the NIHSS within $3 \mathrm{~h}$ from symptom onset [13]. Publicly available, archived, and completely de-identified IMS3 and NINDS IV tPA datasets were obtained from the NINDS, and the study was exempt from Institutional Review Board review.

\section{Study Measures}

All variables had been collected as part of the IMS3 trial and NINDS IV tPA trials. The primary outcome variable for our analysis was good clinical outcome at 3 months defined by the mRS of 0-2 [14]. BI $\geq 95$ was also considered as a measure of good outcome in our study [15]. In both studies, 3-month mRS and BI were determined by a study investigator who was blinded to the treatment assignment and was not involved in 
acute management of the patient. The 24-hour NIHSS examination was performed by a physician who was not involved in initial management. NIHSS documented at $24 \pm 8 \mathrm{~h}$ in both studies was considered as the 24-hour NIHSS score for our analysis. Absolute NIHSS improvement (24-hour NIHSS - baseline NIHSS) and relative NIHSS improvement (24-hour NIHSS - baseline NIHSS/baseline NIHSS) were calculated. The presence of parenchymal hemorrhage $(\mathrm{PH})$ was assessed on a $24 \pm 6 \mathrm{~h}$ non-contrast CT scan and classified as PH1 or PH2 in IMS3 [12]. Additional imaging variables such as infarct volume at follow-up were not available in the publicly available datasets for analysis. EQ5D ${ }^{\mathrm{TM}}$ questionnaires were completed by IMS3 participants at 3 months. Using published utility weights for a United States population, an EQ5D index score was calculated for each participant [16]. For the US general population, the possible EQ-5D index scores range from -0.11 to 1.0 on a scale where 0.0 equals death and 1.0 represents perfect health. Patients who died prior to 3 months were excluded from the analysis and no data were imputed.

\section{Statistical Analysis}

The correlation between two variables was assessed using the Pearson's coefficient. Receiver operator characteristic curve analysis was performed to determine the predictive power [area under the curve (AUC)] of the 24-hour NIHSS, absolute and relative NIHSS improvement for 3-month mRS 0-2 and for 3-month BI $\geq 95$. Multivariable logistic regression was used to model mRS 0-2 at 3 months. Missing data points (3-month mRS, BI, and 24-hour NIHSS) were not imputed in either database. SPSS Version 22 was used for all statistical analyses.

\section{Results}

\section{Patient Characteristics}

Of the 656 patients enrolled in IMS3, 587 were included in the analysis: 69 were excluded due to missing 24-hour NIHSS ( $\mathrm{n}=56$ ) or missing 3-month mRS $(\mathrm{n}=13)$. Patient characteristics of the IMS3 cohort have been previously published [12]. The study population had a mean age of $66 \pm 12$ years, median baseline NIHSS of 17 (IQR 13-20), and mean time-torandomization of $105 \pm 35 \mathrm{~min}$. 48.6\% were randomized to the endovascular arm. PH1 or PH2 occurred in $7.5 \%(n=39)$ of patients and $45.1 \%(n=265)$ achieved a good outcome (mRS $0-2$ ) at 3 months. The 24-hour NIHSS was recorded at a mean time of $22.7 \mathrm{~h}$ (range 16.2531.5), and a median 24-hour NIHSS was 11 (IQR 4-19). BI was available in 509 patients who survived until 3 months. EQ5D data at the 3-month follow-up were available for 423 patients from IMS3.

Validation was performed in the NINDS IV tPA trial cohort, the characteristics of which have been published previously [13]. Of 624 patients enrolled, 619 had both documented 24-hour NIHSS and 3-month mRS available for analysis. The validation cohort had a mean age of $66.9 \pm 11.7$ years, median baseline NIHSS of 15 (IQR 9-20), and mean time-to-randomization of $119 \pm 37 \mathrm{~min}$. The median 24-hour NIHSS was 11 (IQR 4-19) and 44.1\% ( $\mathrm{n}=273$ ) achieved a good outcome (mRS 0-2) at 3 months. All ischemic stroke patients were included for validation regardless of stroke territory. BI was available in 503 patients who survived until 3 months. EQ5D was not available for NINDS IV tPA patients.

\section{Predictive Value of the 24-Hour NIHSS for Long-Term Clinical Outcomes}

NIHSS at $24 \mathrm{~h}$ was strongly positively correlated with 3-month mRS (IMS3: Pearson's coefficient $0.73, \mathrm{p}<0.001$; NINDS tPA: $0.74, \mathrm{p}<0.001$ ), negatively correlated with 3-month BI (IMS3: Pearson's coefficient $-0.64, \mathrm{p}<0.001$; fig. 1 ), as well as inversely correlated with quality of life estimated by the EQ5D index (IMS3: Pearson's coefficient $-0.496, \mathrm{p}<0.001$ ). In contrast, NIHSS at presentation was only weakly to moderately correlated with 3-month mRS (Pearson's coefficient 0.37, p $<0.001$ in IMS3, and 0.54, p < 0.001 in NINDS tPA), BI (IMS3: Pearson's coefficient $-0.29, \mathrm{p}<0.001$ ), and the EQ5D index (IMS3: Pearson's coefficient -0.17 , $p=0.001$ ). These observations corroborate a previous report of high correlation between the 
Rangaraju et al.: Prognostic Value of the 24-Hour Neurological Examination in Anterior Circulation Ischemic Stroke

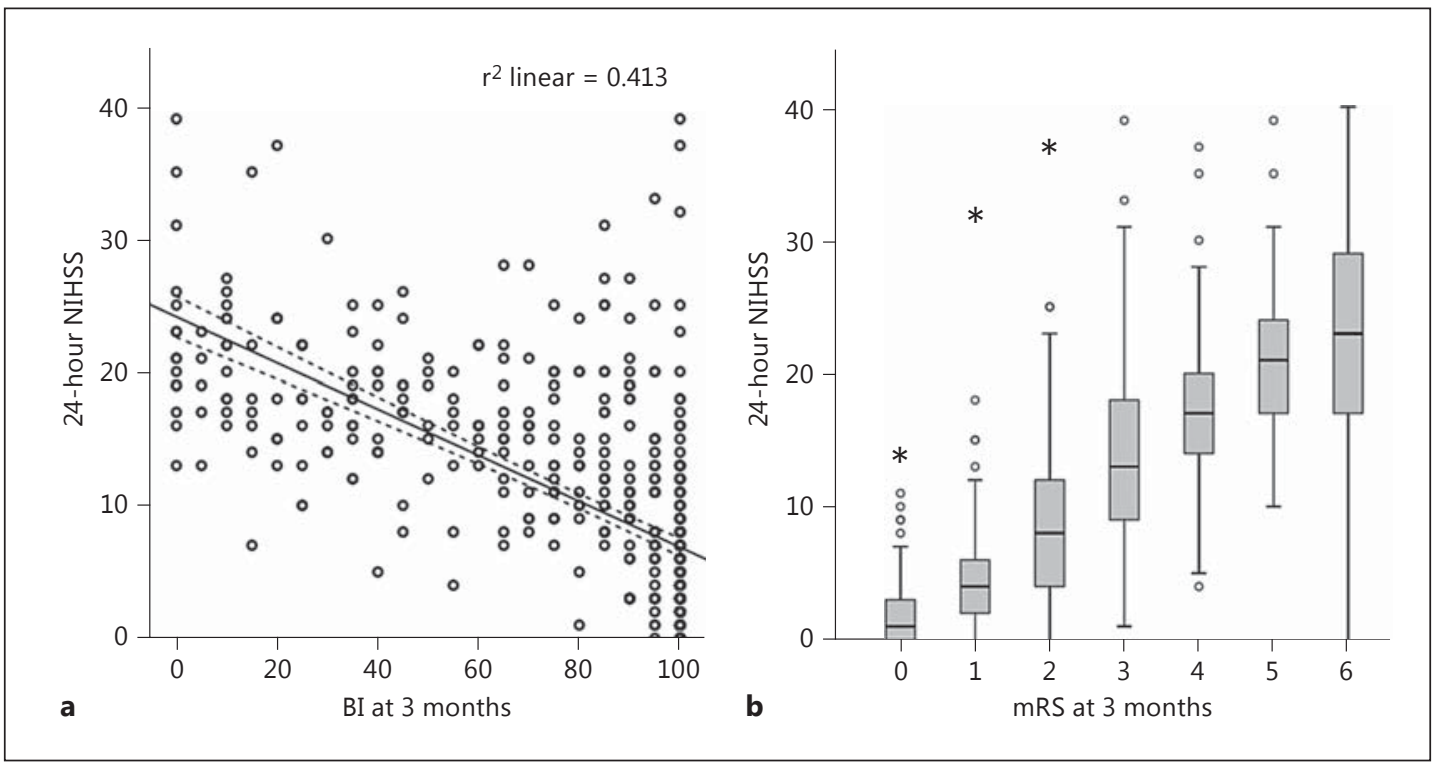

Fig. 1. The 24-hour NIHSS is highly correlated with 3-month clinical disability scores in IMS3. a Negative correlation between the 24-hour NIHSS and 3-month BI. Pearson $\mathrm{R}^{2}$ is shown. Dotted lines represent $95 \%$ confidence limits. b Positive correlation between the 24-hour NIHSS and 3-month mRS. Box plots are shown. Pearson correlation coefficient $=0.73, \mathrm{p}<0.001$.

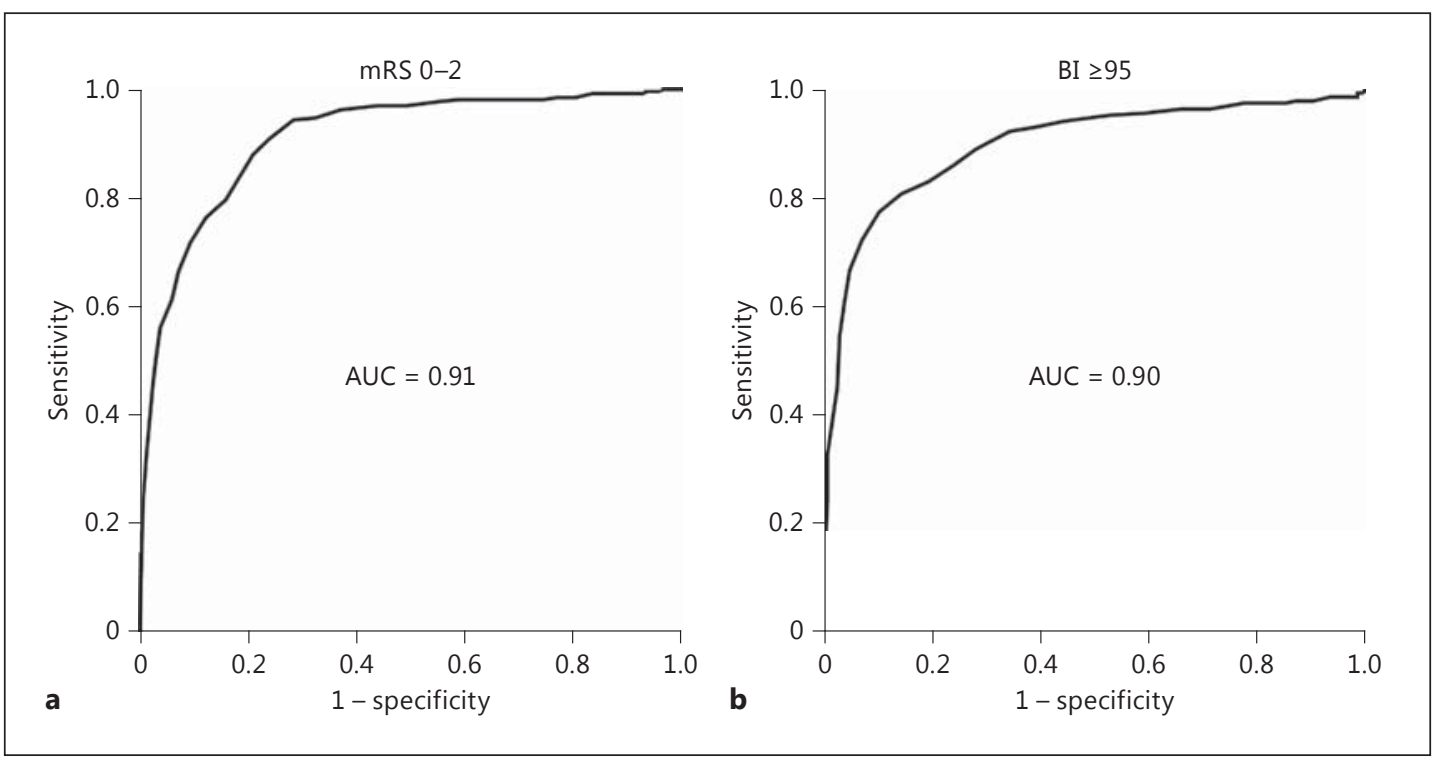

Fig. 2. Excellent predictive power (AUC) of the 24-hour NIHSS for 3-month good clinical outcomes (BI $\geq 95$ and mRS 0-2).

24-hour NIHSS and mRS at 3 months [6]. The predictive power of the 24-hour NIHSS alone for 3-month good outcome (mRS 0-2) was excellent (AUC = 0.92; fig. 2a). The 24-hour NIHSS also had excellent predictive power (AUC $=0.91$ ) for 3-month good outcome defined by BI $\geq 95$; fig. 2b). Similarly, in the NINDS IV tPA cohort, the 24-hour NIHSS had excellent predictive power for 3-month good outcome as defined by mRS 0-2 (AUC $=0.91$ ) and BI $\geq 95$ (AUC = 
Table 1. Comparison of the predictive power of baseline and follow-up NIHSS measurements for 3-month good clinical outcome

\begin{tabular}{lll}
\hline Variable & IMS3 & NINDS tPA \\
\hline Predictive power for 3-month mRS 0-2 & & \\
24-hour NIHSS & 0.91 & 0.922 \\
NIHSS improvement in 24 h (24 h - baseline) & 0.83 & 0.76 \\
Relative improvement in NIHSS over 24 h & 0.87 & 0.83 \\
Baseline NIHSS & 0.73 & 0.78 \\
2-hour NIHSS & n.a. & 0.85 \\
Predictive power for 3-month BI \95 & 0.9 & 0.9 \\
24-hour NIHSS & 0.84 & 0.76 \\
NIHSS improvement in 24 h (24 h - baseline) & 0.87 & 0.83 \\
Relative improvement in NIHSS over 24 h & 0.66 & 0.75 \\
Baseline NIHSS & n.a. & 0.82 \\
2-hour NIHSS & & \\
\hline n.a. = Not available. & & \\
\hline
\end{tabular}

$0.9)$. The 24-hour NIHSS also had excellent predictive power for good clinical outcomes at 6 months (mRS 0-2: AUC $=0.91, \mathrm{BI} \geq 95$ : AUC $=0.89$ ) as well as 12 months (mRS 0-2: AUC = $0.88, \mathrm{BI} \geq 95$ : AUC $=0.87$ ). Absolute and relative change in NIHSS at $24 \mathrm{~h}$ (in IMS3 and NINDS tPA cohorts) as well as 2-hour NIHSS (in the NINDS tPA cohort) had lower predictive powers for good outcome defined by mRS $0-2$ or BI $\geq 95$ as compared to the 24-hour NIHSS (table 1 ). To address the inherent bias of the NIHSS towards left hemispheric involvement, we compared the predictive power of the 24-hour NIHSS for 3-month outcomes in left and right hemispheric strokes separately in IMS3 and found similar results: AUC for 3-month mRS 0-2 was 0.93 in right- and 0.90 in left-sided strokes.

In the multivariable logistic regression analysis using IMS3, age (per year, OR $=0.96,95 \%$ CI 0.94-0.98, $\mathrm{p}<0.01$ ), and the 24-hour NIHSS (per point, OR $=0.75,95 \%$ CI $0.72-0.79, \mathrm{p}<$ 0.001 ) were found to be the only independent predictors of good outcome (3-month mRS $0-2$ ) after adjusting for time to randomization, medical comorbidities (diabetes mellitus, hypertension, atrial fibrillation), race, baseline NIHSS, baseline ASPECTS, treatment arm (endovascular vs. medical), and PH (online suppl. table 1; for all online suppl. material, see www.karger.com/doi/10.1159/000443801) [17]. The correct classification rate of the full 11-variable model was $84.9 \%$, while that of the 2-variable (age, 24-hour NIHSS) model was $83.7 \%$. Dropping age from the two-variable model only slightly reduced the correct classification rate to $82.6 \%$ but significantly impacted the overall model fit (likelihood ratio test statistic comparing the 2-variable to a 1-variable model $=16.06, \mathrm{p}<0.001$ ). Similarly, in the NINDS IV tPA cohort, age (OR $=0.97,95 \%$ CI 0.94-0.99, $\mathrm{p}=0.002)$ and the 24-hour NIHSS $(\mathrm{OR}=0.73,95 \% \mathrm{CI} 0.69-0.76, \mathrm{p}<0.001)$ were the two most important predictors of good outcome after controlling for time to randomization, treatment arm (IV tPA vs. placebo), medical comorbidities, and hemorrhagic transformation. Like in the IMS3, dropping age from the 2-variable model did not affect the correct classification rate (84.3 to $84.7 \%)$, while the model fit was significantly reduced (likelihood ratio test statistic $=11.63, \mathrm{p}<0.001$ ). Although the 24-hour NIHSS may be the more important of the two variables, age still adds additional prognostic value. Also, the observation that baseline NIHSS loses significance as a predictor after including the 24-hour NIHSS confirms that the 24-hour examination is of greater prognostic value than a baseline examination. 
Rangaraju et al.: Prognostic Value of the 24-Hour Neurological Examination in Anterior Circulation Ischemic Stroke

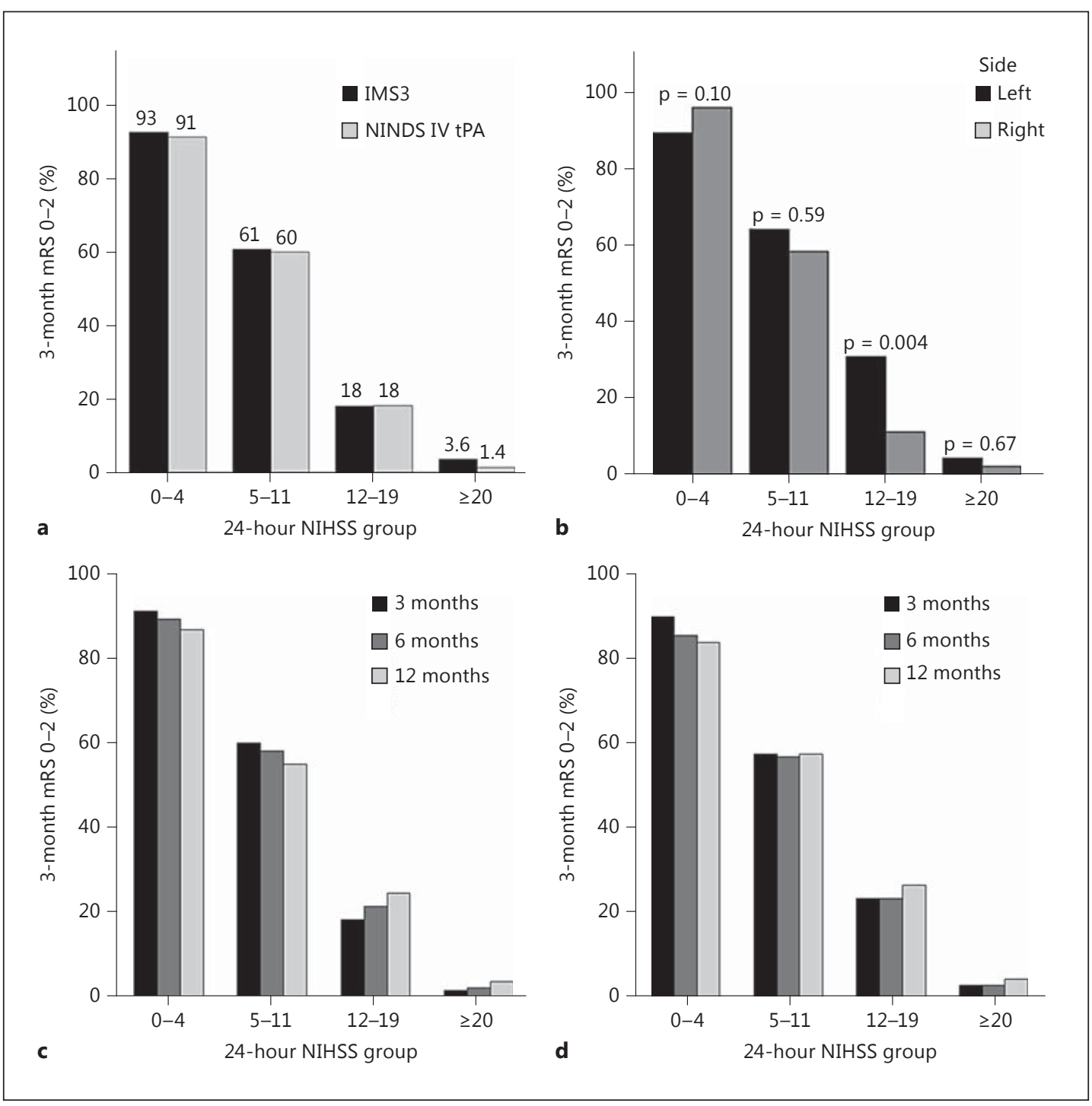

Fig. 3. Risk quartiles based on the 24-hour NIHSS. a Comparison of observed rates of 3-month good outcome (mRS 0-2) in IMS3 and NINDS IV tPA cohorts. b Observed rates of good outcome (mRS 0-2) based on stroke laterality in IMS3 ( $\mathrm{p}$ values calculated based on $\chi^{2}$ or Fisher's exact test are reported). c, d Comparison of 3-, 6-, and 12-month rates of good outcome in the NINDS IV tPA trial across 24-hour NIHSS risk quartiles.

\section{Risk Categories Based on the 24-Hour NIHSS}

Since the 24-hour NIHSS by itself can correctly classify patients as having good or bad outcomes more than $82 \%$ of the time, we created 24 -hour NIHSS risk categories based on observed rates of good outcome (mRS 0-2) in NIHSS quartiles in IMS3 and compared the observations with those in the NINDS IV tPA cohort. In IMS3, patients with a 24-hour NIHSS of $0-4$ had a $93 \%$ chance of achieving a good outcome at 3 months, while patients with a 24 -hour NIHSS of $\geq 20$ had only a $3.6 \%$ chance of achieving a good outcome. Observed rates of good outcomes in the four identified risk categories were very similar in both study cohorts, confirming the validity of this risk stratification (fig. 3a, online suppl. fig. 1). Rates of good clinical outcomes observed at 6 and 12 months in the NINDS IV tPA cohort were also very similar to 3-month outcomes across all 24-hour NIHSS risk quartiles (fig. 3c, d). In IMS3, 
Fig. 4. Comparison of mean EQ5D composite index scores across 24hour NIHSS risk quartiles. Pairwise independent sample $t$ tests (two-tailed) were performed. Patients who did not survive until 3 months were excluded.

\section{DOI: 10.1159/000443801 \\ 2016 S. Karger AG, Base www.karger.com/ine}

Rangaraju et al.: Prognostic Value of the 24-Hour Neurological Examination in Anterior Circulation Ischemic Stroke

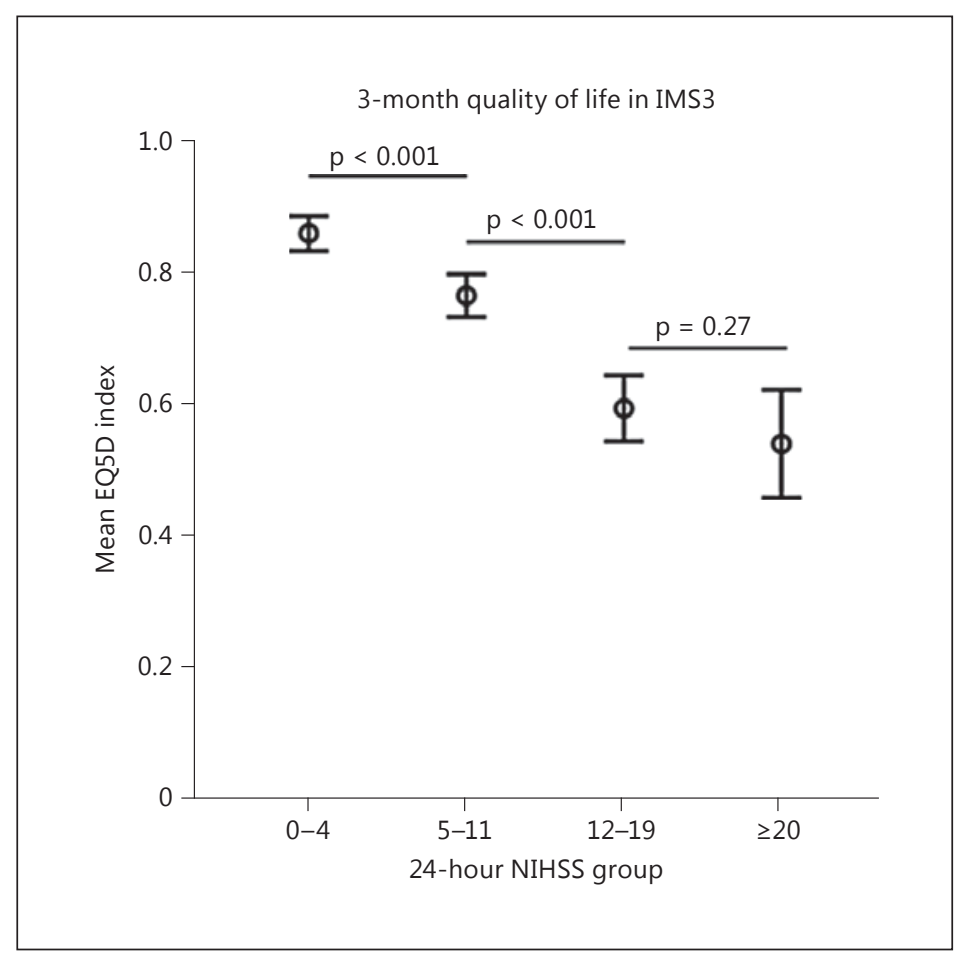

differences in observed rates of good outcome in right and left hemispheric strokes were only observed in the NIHSS $12-19$ subgroup (left: $30.1 \%$ vs. right: $11.2 \%, \mathrm{p}=0.004$; fig. 3b). Similarly, patients in NINDS IV tPA in the 24-hour NIHSS 12-19 group with left-sided strokes had a higher rate of good outcome (23.4\%) as compared to right-sided strokes (13.9\%), although this did not reach statistical significance $(\mathrm{p}=0.13)$. The EQ5D index among IMS3 survivors at 3 months significantly differed among the NIHSS 0-4 (mean index 0.86 \pm 0.16 ), 5-11 (mean index $0.77 \pm 0.18$ ), and $12-19$ (mean index $0.59 \pm 0.26$ ) groups, but was similar in the $12-19$ and $\geq 20$ (mean index $0.52 \pm 0.26$ ) risk groups (fig. 4).

\section{Discussion}

Clinical outcomes in ischemic stroke are typically measured using disability scales such as the mRS and $\mathrm{BI}$, and a 3-month assessment is the most frequently used time point for primary outcome assessment in stroke research. An mRS $0-2$ or BI $\geq 95$ at 3 months is considered a good clinical outcome that is indicative of functional independence in ischemic stroke patients [18]. An early and accurate surrogate of 3-month or later clinical outcomes could facilitate early decision-making, reduce uncertainties regarding prognosis, and also minimize attrition, limit sample size, and reduce costs associated with stroke clinical trials. We show that the 24-hour NIHSS strongly correlates with 3-month outcome and quality of life and has excellent predictive power for 3,6, and 12-month good outcomes in ischemic stroke, specifically those involving the anterior circulation. We also present objective prognostic data and risk groups based on the 24-hour NIHSS that may be clinically meaningful. Based on our results and reports by others [6,7], we propose that the 24-hour NIHSS may represent a very early, yet accurate and reliable surrogate of long-term outcomes in stroke patients. 
Rangaraju et al.: Prognostic Value of the 24-Hour Neurological Examination in Anterior Circulation Ischemic Stroke

The 24-hour poststroke time point is clinically important because many prognostically relevant clinical and radiological variables become available at or after $24 \mathrm{~h}$ following admission for acute ischemic stroke. Patients who received IV tPA typically undergo 24-hour follow-up brain imaging to exclude hemorrhagic transformation, and MRI imaging is often performed around this time point, making an assessment of infarct size and location possible. The risk of hemorrhagic transformation decreases after $24 \mathrm{~h}$, and rapid clinical improvement or early neurological decline is also likely to clear by this time [17]. In addition, discussions regarding prognosis, goals of care and treatment decisions regarding the level of aggressiveness often occur after the first $24 \mathrm{~h}$. Prognostication of clinical outcome at the 24-hour time point should take into consideration all information available at the time of admission as well as the events that have elapsed since admission in order to be prognostically meaningful. A consideration of all possible prestroke and poststroke variables in a predictive model, while ideal, is likely to be impractical, and the complexity of the resulting tool may make it not clinically useful. Instead, it may be possible that a complex variable that is influenced by several measured and unmeasured factors could serve as a single determinant of clinical outcome in ischemic stroke patients. We have found that clinical neurological disability as measured by the 24-hour NIHSS is a robust predictor of 3-month disability with excellent predictive power. Our results from one cohort were replicated with nearly identical results in a second independent cohort, establishing the validity of our observations. The 24-hour NIHSS explained about $50 \%$ of the variance in clinical disability scores at 3 months in both cohorts, consistent with prior observations [6]. Since the NIHSS is universally used and is easily and reliably ascertained [10, 19], its excellent predictive power for 3-month good clinical outcome is particularly remarkable and emphasizes the importance and prognostic value of an objective neurological examination at $24 \mathrm{~h}$ in ischemic stroke patients.

The 24-hour exam is likely influenced by stroke size, stroke location, laterality, and other clinical events such as hemorrhagic transformation and early systemic complications that potentially worsen the neurological examination [20,21]. Therefore, the 24-hour NIHSS likely captures the effect of many other parameters that impact long-term outcomes in stroke patients. Final infarct volume has been proposed as a surrogate of 3-month clinical outcomes and has been found to be a stronger outcome predictor than age, baseline NIHSS, and reperfusion status in the large vessel occlusion population [3]; however, its correlation with 3 -month disability scores is modest (correlation coefficient $0.3-0.5$ ) [22]. Furthermore, the predictive power of previously published multivariate models that incorporate age, final infarct volume, and hemorrhagic complications ranges from 0.75 to $0.85[3,4]$. The 24-hour NIHSS, on the other hand, is strongly correlated with 3-month clinical outcomes and has by itself excellent predictive power (AUC $\approx 0.90$ ) for 3,6 , and 12-month good outcomes defined by the mRS or BI. A direct comparison of the 24-hour NIHSS and infarct volume is needed and whether the addition of the 24-hour NIHSS improves the predictive power and accuracy of previously validated prognostic tools remains to be determined.

Our results also confirm the effect of stroke laterality on clinical outcome. While patients with 24 -hour NIHSS $\leq 11$ and $\geq 20$ had similar outcomes in right and left hemispheric strokes, we observed that patients with a 24-hour NIHSS of 12-19 with left hemispheric strokes had significantly better outcomes compared to right hemispheric strokes despite similar median 24-hour NIHSS scores (15 vs. 16, respectively). We speculate that the higher rate of good outcome in left hemispheric lesions with 24-hour NIHSS 12-19 may be a result of a relatively greater degree of improvement in language dysfunction as compared to subcortical symptoms in stroke patients as well as the bias of the NIHSS towards the dominant hemisphere [21,23]. Despite moderate-to-severe neurological impairment at $24 \mathrm{~h}$ (NIHSS 12-19), 18\% of patients still achieved functional independence at 3 months, suggesting that this group may be a target for aggressive supportive and rehabilitative care. 
Rangaraju et al.: Prognostic Value of the 24-Hour Neurological Examination in Anterior Circulation Ischemic Stroke

A significant strength of our study is the use of large randomized clinical trial databases, where the 24-hour NIHSS examination was performed in a prospective and blinded manner and not calculated retrospectively from documented neurological examinations [12]. Another strength is the replication of observations in two independent trial datasets despite differences between the two trials. IMS3 included patients with higher stroke severity as compared to NINDS IV tPA. All patients in IMS3 were also treated with IV tPA, while half the patients in NINDS IV tPA received placebo. These highlight the greater applicability of our results to anterior circulation stroke patients.

The limitations of our study need to be acknowledged. Firstly, our study did not assess the effect of many clinical, psychological, and socioeconomic factors that also influence stroke outcomes. Accounting for these variables may explain a significant proportion of the variance in mRS not explained by the 24-hour NIHSS. Next, our results are applicable only to anterior circulation stroke since the majority of IMS3 and NINDS IV tPA participants suffered anterior circulation strokes. The validity of the 24-hour NIHSS in posterior circulation strokes needs to be determined. Next, IMS3 participants who did not have a 24-hour NIHSS measurement $(n=26)$ due to chemical paralysis were excluded. Since these represented a minority of the study population, we do not expect their exclusion to bias our results. Missing data for 3-month mRS and BI and 24-hour NIHSS were not imputed because these were the primary variables of interest in our analysis. Although the missing data were minimal, the 3-month BI was available for $78 \%$ in IMS3 and $81 \%$ in NINDS IV tPA trials. We also did not impute all deceased patients as having a BI of 0 in order to limit overestimating the predictive power of the 24-hour NIHSS. Lastly, our analysis also shares the limitations of the mRS as a measure of clinical outcome that is biased towards motor recovery. Therefore, we used BI as an alternative clinical disability measure and EQ5D as a health-related quality-of-life measure. Further validation of the 24-hour NIHSS as a predictor of other quality of life measures (stroke impact scale, SF-36) should be considered in more recent randomized clinical trial data.

In conclusion, we demonstrate that the 24-hour clinical examination using the NIHSS strongly predicts 3-month functional independence and health-related quality of life, and the four 24-hour NIHSS risk quartiles may facilitate accurate and early prognostication in anterior circulation ischemic stroke patients. Our results emphasize the value of consistently capturing the NIHSS at $24 \mathrm{~h}$ in routine clinical practice and also support the use of the 24-hour NIHSS as a surrogate early endpoint in stroke research.

\section{Acknowledgements}

Dr. Rangaraju was partly supported by a training grant (NIH StrokeNET 1U10NS086607-01 PI: Michael Frankel) and is currently a trainee on a T32 grant (2T32 NS 007480-15 PI: Allan I. Levey); Dr. Rangaraju is also partly supported by a clinical research training fellowship from the American Brain Foundation and received a tuition scholarsip from the NIH/Atlanta Clinical and Translational Science Institute (ACTSI) Tuition Scholarship.

\section{Disclosure Statement}

Dr. Jovin reports grant, nonfinancial, and other support from Fundació Ictus Malaltia Vascular, and nonfinancial support from Covidien; personal fees from Silk Road Medical and Air Liquide, and nonfinancial support from Covidien/Medtronic and Stryker Neurovascular outside the submitted work. The other authors report no conflicts of interest. 


\begin{tabular}{l|l}
\hline DOI: $10.1159 / 000443801$ & $\begin{array}{l}\text { C } 2016 \text { S. Karger AG, Basel } \\
\text { www.karger.com/ine }\end{array}$ \\
\hline
\end{tabular}

Rangaraju et al.: Prognostic Value of the 24-Hour Neurological Examination in Anterior Circulation Ischemic Stroke

\section{References}

1 Saposnik G, Cote R, Mamdani M, Raptis S, Thorpe KE, Fang J, Redelmeier DA, Goldstein LB: JURaSSiC: accuracy of clinician vs risk score prediction of ischemic stroke outcomes. Neurology 2013;81:448-455.

2 Saver JL, Fonarow GC, Smith EE, Reeves MJ, Grau-Sepulveda MV, Pan W, Olson DM, Hernandez AF, Peterson ED, Schwamm LH: Time to treatment with intravenous tissue plasminogen activator and outcome from acute ischemic stroke. JAMA 2013;309:2480-2488.

3 Zaidi SF, Aghaebrahim A, Urra X, Jumaa MA, Jankowitz B, Hammer M, Nogueira R, Horowitz M, Reddy V, Jovin TG: Final infarct volume is a stronger predictor of outcome than recanalization in patients with proximal middle cerebral artery occlusion treated with endovascular therapy. Stroke 2012;43:3238-3244.

4 Rangaraju S, Liggins JT, Aghaebrahim A, Streib C, Sun CH, Gupta R, Nogueira R, Frankel M, Mlynash M, Lansberg $\mathrm{M}$, et al: Pittsburgh outcomes after stroke thrombectomy score predicts outcomes after endovascular therapy for anterior circulation large vessel occlusions. Stroke 2014;45:2298-2304.

5 Broderick JP, Lu M, Kothari R, Levine SR, Lyden PD, Haley EC, Brott TG, Grotta J, Tilley BC, Marler JR, et al: Finding the most powerful measures of the effectiveness of tissue plasminogen activator in the NINDS tPA stroke trial. Stroke 2000;31:2335-2341.

6 Saver JL, Altman H: Relationship between neurologic deficit severity and final functional outcome shifts and strengthens during first hours after onset. Stroke 2012;43:1537-1541.

7 Brown DL, Johnston KC, Wagner DP, Haley EC Jr: Predicting major neurological improvement with intravenous recombinant tissue plasminogen activator treatment of stroke. Stroke 2004;35:147-150.

8 Brott T, Adams HP Jr, Olinger CP, Marler JR, Barsan WG, Biller J, Spilker J, Holleran R, Eberle R, Hertzberg V, et al: Measurements of acute cerebral infarction: a clinical examination scale. Stroke 1989;20:864-870.

9 Lyden P, Brott T, Tilley B, Welch KM, Mascha EJ, Levine S, Haley EC, Grotta J, Marler J: Improved reliability of the NIH Stroke Scale using video training. NINDS TPA Stroke Study Group. Stroke 1994;25:2220-2226.

10 Goldstein LB, Samsa GP: Reliability of the National Institutes of Health Stroke Scale. Extension to non-neurologists in the context of a clinical trial. Stroke 1997;28:307-310.

11 Nagaraja N, Warach S, Hsia AW, Adams HP Jr, Auh S, Latour LL, Merino JG: Association between neurologic improvement with decline in blood pressure and recanalization in stroke. JAMA Neurol 2014;71:1555-1558.

12 Broderick JP, Palesch YY, Demchuk AM, Yeatts SD, Khatri P, Hill MD, Jauch EC, Jovin TG, Yan B, Silver FL, et al: Endovascular therapy after intravenous t-PA versus t-PA alone for stroke. N Engl J Med 2013;368:893-903.

13 Tissue plasminogen activator for acute ischemic stroke. The National Institute of Neurological Disorders and Stroke rt-PA stroke study group. N Engl J Med 1995;333:1581-1587.

14 Bonita R, Beaglehole R: Recovery of motor function after stroke. Stroke 1988;19:1497-1500.

15 Quinn TJ, Langhorne P, Stott DJ: Barthel index for stroke trials: development, properties, and application. Stroke 2011;42:1146-1151.

16 Shaw JW, Johnson JA, Coons SJ: US valuation of the EQ-5D health states: development and testing of the D1 valuation model. Med Care 2005;43:203-220.

17 Fiorelli M, Bastianello S, von Kummer R, del Zoppo GJ, Larrue V, Lesaffre E, Ringleb AP, Lorenzano S, Manelfe C, Bozzao L: Hemorrhagic transformation within 36 hours of a cerebral infarct: relationships with early clinical deterioration and 3-month outcome in the European Cooperative Acute Stroke Study I (ECASS I) cohort. Stroke 1999;30:2280-2284.

18 Uyttenboogaart M, Stewart RE, Vroomen PC, De Keyser J, Luijckx GJ: Optimizing cutoff scores for the Barthel index and the modified Rankin scale for defining outcome in acute stroke trials. Stroke 2005;36:1984-1987.

19 Goldstein LB, Bertels C, Davis JN: Interrater reliability of the NIH Stroke Scale. Arch Neurol 1989;46:660-662.

20 Tong DC, Yenari MA, Albers GW, O’Brien M, Marks MP, Moseley ME: Correlation of perfusion- and diffusionweighted MRI with NIHSS score in acute (<6.5 hour) ischemic stroke. Neurology 1998;50:864-870.

21 Fink JN, Selim MH, Kumar S, Silver B, Linfante I, Caplan LR, Schlaug G: Is the association of National Institutes of Health Stroke Scale scores and acute magnetic resonance imaging stroke volume equal for patients with right- and left-hemisphere ischemic stroke? Stroke 2002;33:954-958.

22 Saver JL, Johnston KC, Homer D, Wityk R, Koroshetz W, Truskowski LL, Haley EC: Infarct volume as a surrogate or auxiliary outcome measure in ischemic stroke clinical trials. The RANTTAS investigators. Stroke 1999;30: 293-298.

23 Lazar RM, Minzer B, Antoniello D, Festa JR, Krakauer JW, Marshall RS: Improvement in aphasia scores after stroke is well predicted by initial severity. Stroke 2010;41:1485-1488. 